\section{Racial Disparities in Medication Use: JMCP's Response and Call for Submissions}

Like many individuals and organizations, we at the Journal of Managed Care+Specialty Pharmacy (JMCP) have been grappling with the reality of racial injustice. We have asked ourselves, what role do we have in the problem? How can we be part of the solution?

The answer is simple: we don't know. There is no guide for how peer-reviewed journals should address these issues.

But we do know this: Our mission at JMCP is "to advance evidence-based decision making to improve patient health." We make no caveats to improving patient health. We mean all patients-all ages, gender identities, communities, cultures, ethnicities, and races-irrespective of whether they currently have benefits. That means addressing racial injustice is squarely within our mission, especially as it relates to evidence-based decisions that affect patient health.

Dr. Stephen Kogut's Viewpoints article in this month's issue of JMCP, "Racial Disparities in Medication Use: Imperatives for Managed Care Pharmacy," is a call to action for managed care pharmacy to address racial injustice in our businesses. ${ }^{1}$ The first time we read it, it made some of us a bit uncomfortable. However, we think that is a good thing. We should challenge ourselves to examine our profession through different lenses, particularly those that are supported with evidence, as in Dr. Kogut's viewpoint.*

As Dr. Kogut recommends in his article, the team at JMCP acknowledges that there is evidence of racial disparities in medication use systems. The link between racism and inequities in patient health is irrefutable. We are writing our own playbook on how our journal can contribute to reducing these inequities. As such, we have assembled a work group at JMCP to investigate and take action. We have identified 2 guiding principles as we set out on this work:

We have adopted a position of learning. Our editorial advisory board and editorial team lack sufficient diversity from underrepresented minority populations. As the adage goes, "We don't know what we don't know." So, we are learning. We are listening. We are reading. We are meeting with experts. We are beginning a journey.

We are committed to the sustained efforts required to influence change. We are not publishing this letter to "check the box" and compliment ourselves for our contribution. Rather, we commit to continuously review our organizational structure and policies to eliminate inequities in all aspects of our work. We commit to working to publish papers on the subject of racial injustice in medication use and to increase the racial sensitivity of all papers we publish.

We have already taken some action steps at the journal. For example, we are actively seeking to increase the diversity of our editorial advisory board, which is a volunteer board comprised of individuals with experience in managed care pharmacy research and who have served as a peer reviewer for JMCP. Interested individuals can apply each fall through the AMCP website, or contact Editor-in-Chief Laura Happe for more information. Further, we have updated our style guide in response to a change in the AMA Manual of Style related to capitalization of racial and ethnic categories.

\section{CALL FOR SUBMISSIONS}

In his essay, Dr. Kogut calls for responses to his article, acknowledging that many people have been working to reduce racial disparities in medication use for years. The editors of JMCP would like to underscore and expand upon his call.

We invite you to submit your papers and help us to continue this conversation. We welcome research articles, particularly those that evaluate interventions that attempt to reduce disparities. We also recommend foundational research studies that can be used to design effective interventions. We welcome commentaries (i.e., viewpoints). How is your organization tackling this problem? What are the deficiencies in our research methods and clinical protocols as they relate to race? Finally, we welcome letters to the editor that comment either on a published paper or on aspects of racial injustice related to managed care pharmacy.

As an example of relevant research, Li et al. (2020) explored the effect of a Medicare reimbursement policy change on the use of erythropoiesis-stimulating agents (ESAs) and blood transfusions in this month's issue. ${ }^{2}$ The policy had the intended effect of reducing ESA use by $50 \%$ in all race and ethnicity groups. However, there was an unintended effect of increasing blood transfusions, which was of a higher magnitude in the Black population (50\%) compared with the full study cohort (10\%).

Finally, and importantly, we acknowledge that we are not focusing on racial disparities to the exclusion of other communities that suffer from discrimination. We hope that, by focusing on race, we can improve equity across the spectrum. Submissions related to all areas of discrimination are encouraged. 


\section{CONCLUSIONS}

JMCP welcomes engagement from individuals of all demographics and backgrounds in the form of submitters, peer reviewers, board volunteers, and readers.

JMCP invites submission of research articles and commentaries on racial disparities and related interventions in the medication use system.

Please join us in acting against racial injustices.

Laura E. Happe, PharmD, MPH

Editor-in-Chief, JMCP

University of Florida

laurahappe@jmcp.org

\section{Donald G. Klepser, PhD, MBA}

Associate Editor, JMCP

University of Nebraska Medical Center

Carrie McAdam-Marx, BSPharm, MS, PhD

Section Editor, JMCP

University of Nebraska Medical Center

\section{Melissa S. McCart, PharmD, MS}

Associate Editor, JMCP

Xcenda

\section{Karen L. Rascati, PhD}

Associate Editor, JMCP

University of Texas College of Pharmacy

\section{Michael T. Rupp, BSPharm, PhD}

Section Editor, JMCP

Midwestern University

\section{WORK GROUP MEMBERS}

\section{Bonnie Greenwood, PharmD, BCPS}

Chair, Editorial Advisory Board, JMCP

Commonwealth Medicine, University of Massachusetts

Medical School

\section{Prabashni Reddy, BSPharm}

Editorial Advisory Board, JMCP

Partners Healthcare

Patty Taddei-Allen, PharmD, MBA, BCACP, BCGP

Editorial Advisory Board, JMCP

WellDyne

\section{Manish Mittal, PhD}

Editorial Advisory Board, JMCP

AbbVie

\section{Patti Peeples, PhD, RPH}

Editorial Advisory Board, JMCP

Healtheconomics.com

\section{Craig S. Stern, MBA, PharmD, RPh}

Editorial Advisory Board, JMCP

ProPharma Pharmaceutical Consultants

\section{DISCLOSURES}

No funding supported the writing of this letter. The authors have nothing to disclose.

\section{REFERENCES}

1. Kogut S. Racial disparities in medication use: imperatives for managed care pharmacy. J Manag Care Spec Pharm. 2020;26(11):1468-74.

2. Li M, Schulz R, Chisholm-Burns M, Wang J, Lu ZK. Racial/ ethnic and gender disparities in the use of erythropoiesis-stimulating agents and blood transfusions: cancer management under Medicare's reimbursement policy. J Manag Care Spec Pharm. 2020;26(11):1477-86. 